\title{
Valoração econômica da área rural de coxilha rica, campos naturais de Lages/SC
}

A região da Coxilha Rica localiza-se na zona rural do município de Lages/SC e é conhecida pela beleza de seus recursos naturais e sua relevância na história e cultura da serra catarinense, porém, vem sendo ameaçada pela intensificação da exploração agropecuária e florestal. Tal fato motivou a realização do presente estudo com o objetivo de estimar o valor ambiental desta região, a partir da mensuração da disposição a pagar (DAP) da população local pela sua preservação. Utilizouse o Método de Valoração Contingente (MVC) durante os meses de abril a julho de 2016, onde foram entrevistadas 252 pessoas residentes em Lages e questionadas, via método referendo, se aceitam contribuir financeiramente para a preservação da Coxilha Rica. O perfil socioeconômico dos respondentes e uma Escala de Percepção Ambiental (EPA) foram utilizados como variáveis explicativas. As variáveis Renda, Preço e EPA foram significativas na estimativa dos modelos logit e probit, resultando um valor ambiental individual de $\mathrm{R} \$ 25,81$ e $\mathrm{R} \$ 25,17$ mensais, respectivamente. Concluiu-se que os votos de protestos comprometem significativamente os resultados dos modelos e que a variável EPA se mostrou promissora nos estudos de valoração ambiental.

\section{Economic valoration of the rural area of coxilha rica, Lages/SC natural fields}

\begin{abstract}
The region of Coxilha Rica is located at the countryside of Lages/SC and known by the beauty of its natural resources and its relevance in the history and culture of Santa Catarina, however, is being threatened by the intensification of agriculture and forestry. This fact motivated the present study aimed to estimate the environmental value of this area, from the Measurement of Willingness to Pay (WTP) of the local population for its preservation. We used the Contingent Valuation Method (CVM) during the months from April to July 2016, where 252 people living in Lages interviewed and questioned by referendum method, they accepted to contribute financially to the preservation of Coxilha Rica. The socioeconomic profile of respondents and an Environmental Concern Scale (EPA) used as explanatory variables. The variables Income, Price and EPA were significant in the estimation of logit and probit models, resulting in an individual environmental value of R\$ 25.81 and $R \$ 25.17$ per month, respectively. We concluded that the votes of protests significantly compromise the results of the models and the EPA variable proved to be promising in the study of environmental valuation.
\end{abstract}

Keywords: Contingent Valuation Method; Willingness to Pay; Environmental Concern Scale.

Topic: Valoração e Economia Ambiental

Reviewed anonymously in the process of blind peer.
Received: 10/04/2018

Approved: 24/05/2018
Flávio José Simioni (iD

Universidade do Estado de Santa Catarina, Brasil

http://lattes.cnpq.br/2737272474496684

http://orcid.org/0000-0002-6392-5073

flavio.simioni@udesc.br

\section{Carolina Donadel (iD)}

Universidade do Estado de Santa Catarina, Brasil

http://lattes.cnpq.br/7494323510834984

http://orcid.org/0000-0001-6127-3959

cdonadel1@gmail.com

Julia Nercolini Göde (1D)

Universidade do Estado de Santa Catarina, Brasil

http://lattes.cnpq.br/8172218911058696

http://orcid.org/0000-0003-4336-0877

julianercolini@hotmail.com

\section{Ana Caroline Cantarelli \\ Universidade do Estado de Santa Catarina, Brasil \\ http://lattes.cnpq.br/4465399598955887 \\ ana-cantarelli@outlook.com}

\section{Lais Sartori}

Universidade do Estado de Santa Catarina, Brasil

http://lattes.cnpq.br/6792613125642350

lais.sartori@hotmail.com
Referencing this:

SIMIONI, F. J.; DONADEL, C.; GÖDE, J. N.; CANTARELLI, A. C.; SARTORI, L.. Valoração econômica da área rural de coxilha rica, campos naturais de Lages/SC. Revista Ibero Americana de Ciências Ambientais, v.9, n.4, p.393-406, 2018. DOI: http://doi.org/10.6008/CBPC21796858.2018.004.0031

DOI: 10.6008/CBPC2179-6858.2018.004.0031 


\section{INTRODUÇÃO}

O acelerado crescimento urbano, a expansão agrícola e a consequente alteração da paisagem e das características ambientais que estes dois processos causam têm gerado uma série de problemas ambientais no Brasil. Sobre a expansão agrícola, Teixeira (2005) afirma que o espaço agrário brasileiro passou por grandes mudanças a partir dos anos 50 até os dias atuais. Na década de 1950 iniciou-se o processo de modernização da agricultura, que se acentuou a partir da década de 1960, principalmente nas regiões Sul e Sudeste e expandiu-se para as demais regiões do país.

Motter (2015) avaliando a transformação da paisagem no noroeste do RS, contatou que a modernização rural no período recente intensificou a intervenção no espaço agrário, aprofundando os desequilíbrios ambientais. Bolsan (2006) destaca a destruição das florestas, queda da biodiversidade, erosão dos solos e contaminação do ambiente e dos alimentos são os principais problemas ambientais, associados, em grande medida, ao monocultivo e à intensificação do uso de máquinas e insumos químicos.

Um segundo efeito associado à modernização foi o avanço do uso da terra sobre novas fronteiras agrícolas, sobretudo para as regiões Centro-Oeste e Norte do país. Neste contexto, a região da Coxilha Rica também despertou interesse dos produtores de grãos e tem atraído significativos investimentos, cujo potencial de exploração pode chegar a 40 mil hectares. A Coxilha Rica é uma região situada na zona rural dos municípios de Lages e Capão Alto, com área de 1.136,5 Km² e 244 km de perímetro. Esta região localiza-se a mais de 900 metros acima do nível do mar e é a maior área de campos naturais com matas de araucária, rios e riachos preservados de Santa Catarina.

Este modelo de agricultura mecanizada e com uso abusivo de insumos tem o potencial de causar impactos ambientais ainda inexistentes na região da Coxilha Rica, até então ocupada essencialmente com a produção pecuária de corte extensiva. A alteração da paisagem, dos habitats da fauna local, da vazão dos rios da região e a redução da biodiversidade da localidade onde passa o Caminho das Tropas, que foi muito importante durante o ciclo do tropeirismo, no século XVIII, configuram-se nas principais ameaças à Coxilha Rica, que possui grande importância histórica e cultural para a região de Lages (POLÊSE, 2014). Ainda de acordo com Polêse (2014; 2015), a Coxilha Rica é um dos últimos locais ainda conservados e que oferece as condições necessárias para a potencial expansão da fronteira agroflorestal do estado. Além disso, possui grande potencial hídrico de significativa importância para a região, o que fez com que a região seja considerada para a implantação de novas hidrelétricas. Outra ameaça considerável ao local é o possível aproveitamento energético devido ao potencial eólico que possui pela presença de ventos em seus campos naturais. Diante da problemática exposta, o objetivo do presente trabalho foi estimar o valor ambiental dado pela população de Lages pela preservação da Coxilha Rica, com a utilização do Método de Valoração Contingente (MVC) em combinação com o uso da Escala de Percepção Ambiental (EPA).

\section{MATERIAIS E MÉTODOS}

\section{Área Estudada}


A pesquisa foi realizada no município de Lages/SC (região serrana do Sul do Brasil) e o recurso natural estudado foi a área rural denominada Coxilha Rica (Figura 1). A região da Coxilha Rica localiza-se na zona rural do município de Lages/SC e é caracterizada pelas suas amplas planícies onduladas ocupadas por campos naturais e fragmentos de matas de Araucárias. A importância da Coxilha Rica deve-se, em grande parte, à beleza de seus recursos naturais e sua relevância na história e cultura da serra catarinense, devido ao fato de ser cruzada pelo Caminho das Tropas, via utilizada pelos tropeiros no século XVIII para transporte de gado do Rio Grande do Sul ao Sudeste do Brasil, atividade responsável por desenvolver o comércio e a economia da região. Tais características são utilizadas como atrativos turísticos, envolvendo passeios em fazendas de turismo rural, turismo de aventura, equestre entre outras. As exuberantes paisagens naturais da Coxilha Rica e os remanescentes dos caminhos das tropas vêm sendo ameaçados desde o século passado até os dias atuais pela intensificação da exploração agropecuária e introdução de espécies florestais exóticas. Desta forma, levando-se em conta a fragilidade dos ecossistemas e as ameaças com as quais estes vem sofrendo, é recorrente o tema relacionado à preservação ambiental da Coxilha Rica.

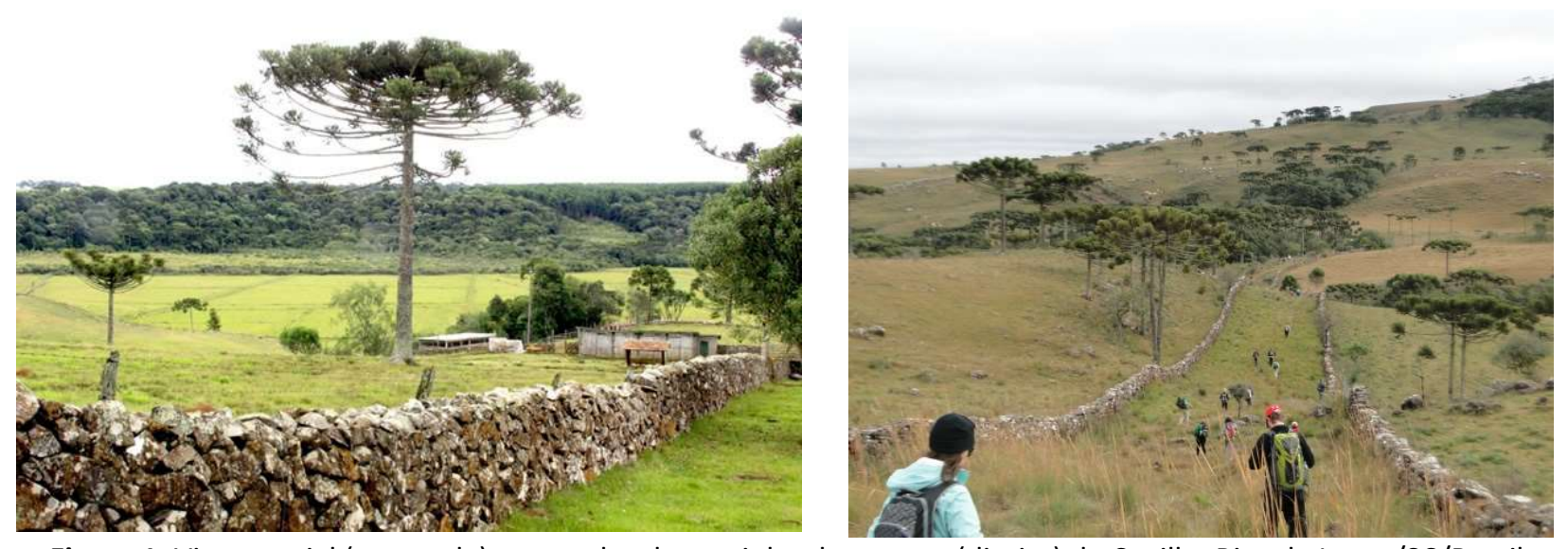

Figura 1: Vista parcial (esquerda) e corredor do caminho das tropas (direita) da Coxilha Rica de Lages/SC/Brasil. Fonte: IPHAN (2012) e TREKKING (2013).

\section{População e Amostra}

A população investigada foi restrita aos residentes no município de Lages, por no mínimo cinco anos, com idade superior a 16 anos e que possuíam renda. De acordo com o IBGE (2016) a população estimada do município de Lages foi de 158.732 habitantes em 2015. O tamanho da amostra foi definido considerando um erro máximo de $6 \%$ ao nível de confiança de $95 \%$, com estimativa da proporção $p$ a partir de uma pré-amostra (SILVA et al., 1997). Assim, foram entrevistadas 252 pessoas residentes em Lages, durante o período de abril a julho de 2016, de forma aleatória e proporcional, considerando a estratificação da população por bairros da cidade.

\section{Estratégia de coleta dos dados}

Com o objetivo de representar adequadamente a percepção da população em relação ao valor do recurso natural investigado, a equipe de investigadores responsáveis pela aplicação do questionário foi capacitada e, além disso, obteve aprendizado sobre a aplicação do método resultante de estudos anteriores. 
O instrumento de coleta de dados foi um questionário, cuja estruturação foi realizada com base nos trabalhos de Freitas et al. (2010), Gullo (2010) e Oliveira et al. (2013). Foram observadas as recomendações de Arrow et al. (1993) para a minimização dos vieses causados pelo desenho do questionário e da amostra.

Para a valoração ambiental proposta neste estudo, foi utilizado o MVC, com base na DAP das pessoas para a preservação da área da Coxilha Rica, constituindo-se na variável dependente. Para a identificação da DAP, o primeiro procedimento adotado nas entrevistas foi a apresentação das principais características do recurso ambiental estudado, procurando demonstrar o cenário que envolve a pesquisa. Feito isso, o entrevistado foi questionado sobre seu conhecimento sobre a Coxilha Rica e sobre seu perfil socioeconômico. Na sequência, foi questionado se aceita ou rejeita contribuir financeiramente para a preservação do recurso ambiental pesquisado, considerando o método referendum conforme Arrow et al. (1993). As opções de preço a pagar sugeridas aos entrevistados foram $R \$ 1,00, R \$ 2,50, R \$ 5,00, R \$ 7,50, R \$ 10,00, R \$ 15,00$ e $R \$ 20,00$, definidos previamente a partir de respostas abertas de uma amostragem piloto usada para identificar os parâmetros monetários. Nos casos em que a resposta foi negativa, o entrevistado foi questionado sobre qual seria o valor máximo que ele estaria disposto a contribuir. Se a resposta era zero, buscou-se identificar o motivo para verificar se reflete uma incapacidade de pagamento ou um 'voto de protesto', buscando adequar a resposta encontrada ao escopo, conforme sugere Desvousges et al. (2012). Na etapa final, o entrevistado respondeu a um conjunto de 15 assertivas que representam a Escala de Percepção Ambiental (EPA).

O MVC é amplamente utilizado para identificar o valor de bens e serviços ambientais, obtido via aplicação de questionários em que se avalia a preferência das pessoas (MITCHELL et al., 1989). Para a determinação da disposição a pagar, são comumente utilizados modelos econométricos que consideram as características do perfil do entrevistado, tais como variáveis socioeconômicas e outras informações relacionadas ao bem avaliado (SILVA et al., 2004; MAJUMDAR et al., 2011; OLIVEIRA et al., 2013; LO et al., 2015).

\section{Modelo de análise}

Para a identificação do valor dado ao recurso ambiental, os dados foram analisados preliminarmente utilizando-se de estatísticas descritivas. Posteriormente, também foram submetidos à construção dos modelos de regressão logística logit e probit que permitem realizar predição para variáveis dependentes dicotômicas a partir de um conjunto de variáveis explicativas.

Com os modelos de regressão foi possível identificar quais variáveis aumentam a probabilidade de os indivíduos contribuírem para a preservação da Coxilha Rica. As estimativas de probabilidade situam-se dentro do intervalo zero e um e, como a estimação não é linear, dado que o efeito marginal de uma variável sobre a probabilidade depende do comportamento de outras variáveis. Os modelos de regressão logit e probit são especificados pelas equações 1 e 2 :

$$
\begin{array}{ll}
\text { Equação 1= } & \text { Logit }: p=\operatorname{Pr}[Y=1]=\frac{1}{1+e^{-X^{\prime} \beta}} \\
\text { Equação 2 } & \text { Probit }: p=\operatorname{Pr}[Y=1]=\int_{-\infty}^{X^{\prime} \beta} \frac{1}{\sqrt{2 \pi}} e^{\frac{1}{2} z^{2}} d z=N\left(X^{\prime} \beta\right)
\end{array}
$$


Onde, Y é a variável dependente e indica se o indivíduo concorda ou não com o valor da DAP proposto pelo método referendo ( $0=$ não; $1=s i m$ ), X é um conjunto de variáveis independentes (parâmetros) que influenciam, Pr é a probabilidade de o indivíduo apresentar DAP positiva e, $\beta$ são os coeficientes dos parâmetros estimados. As variáveis independentes inicialmente utilizadas no modelo e suas respectivas hipóteses foram:

Conhece: é uma variável categórica, assumindo 0 (zero) quando o entrevistado não conhece a Coxilha Rica e 1 (um) quando ele conhece. Espera se um efeito positivo, ou seja, quanto o entrevistado conhece o recurso ambiental terá maior percepção quanto ao valor ambiental, histórico e cultural para a região, tendo como consequência uma maior probabilidade de concordar em contribuir com o valor sugerido.

Idade: representa a idade, em anos, da pessoa entrevistada. Espera-se um efeito positivo desta variável, ou seja, as pessoas com maior idade possuem uma trajetória de vida e estabilidade financeira que aumentam a probabilidade de contribuir com uma DAP.

Educação: a variável foi medida considerando o nível de escolaridade do entrevistado, atribuindo-se 0 (zero) para os casos em que o respondente não estudou, 1(um) para os que apresentaram ensino fundamental incompleto, 2 (dois) para ensino fundamental completo, 3 (três) para ensino médio incompleto, 4 (quatro) para ensino médio completo, 5 (cinco) para ensino superior incompleto, 6 (seis) para ensino superior completo, 7 (sete) para aqueles com pós-graduação incompleta, 8 (oito) para mestrado e 9 (nove) para doutorado. Espera-se um efeito positivo desta variável, indicando que um nível escolar mais elevado melhora a compreensão da necessidade da preservação ambiental, aumentando assim a probabilidade de apresentar uma DAP positiva.

Renda: representa a renda mensal familiar bruta, atribuindo-se 0 (zero) para os casos em que o respondente não possui renda, 1 (um) para as famílias com renda de até 1 salário mínimo (SM), 2 (dois) para renda de um a dois SM, 3 (três) para renda de dois a cinco SM, 4 (quatro) para renda de cinco a dez SM, 5 (cinco) para renda de dez a 20 SM, 6 (seis) para renda de 20 a 30 SM e 7 (sete) para as famílias com renda superior a 30 SM. Espera-se um efeito positivo, indicando que um nível de renda mais elevado aumenta a probabilidade de contribuir com uma DAP positiva.

Pessoas: representa o número de pessoas da família do entrevistado. Espera-se um efeito positivo sobre a aceitação de contribuir com o valor sugerido, em função de que mais pessoas usufruem do benefício gerado e pelo aumento da renda familiar.

EPA - Escala de Percepção Ambiental: foi obtida a partir de um conjunto de 15 assertivas, algumas positivas e outras negativas, tendo como resposta uma escala de 5 a 1, onde: 5 (Concorda totalmente); 4 (Concorda); 3 (Indiferente); 2 (Discorda) e 1 (Discorda totalmente) (Tabela 1). A escala apresenta resultados que podem variar de 15 a 75 pontos, sendo que quanto maior a consciência ambiental, maior é a EPA. Para a obtenção da pontuação do entrevistado, as respostas das assertivas negativas (questões 2, 4, 5, 8, 9, 11, 12 e 13) foram invertidas, ou seja, a resposta 1 passou a ser 5, 2 passou a ser 4 e assim, sucessivamente. Tal como com a escolaridade, espera-se um efeito positivo, dado que a maior consciência/preocupação do indivíduo com as questões ambientais, maior é a probabilidade de concordar em contribuir com o valor sugerido. 
Preço: valor de $\mathrm{R} \$ 1,00, \mathrm{R} \$ 2,50, \mathrm{R} \$ 5,00, \mathrm{R} \$ 7,50, \mathrm{R} \$ 10,00, \mathrm{R} \$ 15,00$ e $\mathrm{R} \$ 20,00$ apresentado ao entrevistado pelo método referendum. Espera um efeito negativo, ou seja, quanto maior for o preço sugerido, menor é a probabilidade de o indivíduo apresentar resposta afirmativa.

Os modelos de regressão logit e probit foram realizados considerando todas as variáveis independentes, inicialmente com os votos de protestos (Modelo 1) e, posteriormente, sem os votos de protestos (Modelo 2). O modelo final foi realizado sem os votos de protesto e mantendo-se somente as variáveis que foram significativas (Modelo 3). As regressões foram realizadas no software Minitab Statistical Software (MINITAB, 2009).

Tabela 1: Assertivas para a obtenção da Escala de Percepção Ambiental - EPA.

\begin{tabular}{|ll|}
\hline 1. & O governo tem que introduzir medidas drásticas para parar com a poluição uma vez que poucas pessoas respeitam as leis. \\
\hline 2. & Não deveríamos nos preocupar em matar animais de caça, pois no futuro as coisas se equilibram. \\
\hline 3. & Você estaria disposto (a) a fazer sacrifícios pessoais para reduzir o ritmo da poluição mesmo que os resultados imediatos \\
pareçam pouco significantes.
\end{tabular}
Fonte: adaptação do texto de García (2000).

\section{RESULTADOS E DISCUSSÃO}

Os resultados da pesquisa indicaram que $89,7 \%$ dos entrevistados conheciam ou ouviram falar da Coxilha Rica, $60,3 \%$ eram do sexo feminino e $40,7 \%$ do sexo masculino. De acordo com o perfil dos questionados, poucos possuem formação escolar completa, ou seja, uma parcela significativa dos entrevistados apresentou baixo nível de escolaridade, visto que $83,3 \%$ não chegaram a concluir o ensino médio. A falta de investimento ou oportunidade na formação educacional dos entrevistados, indicam consequências perceptíveis em suas rendas, no qual 15\% possuem renda familiar mensal até um salário mínimo, 38,1\% possuem renda entre um e dois salários mínimos mensais, 30,6\% entre dois e cinco salários mínimos, 11,5\% entre 5 e 10 salários mínimos e 4,8\% com mais de dez salários mínimos mensais.

A análise descritiva dos dados revelou que $61,1 \%$ dos entrevistados estiveram dispostos a contribuir com o valor sugerido, enquanto $38,9 \%$ apresentaram resposta zero. Dentre os entrevistados que responderam de maneira negativa, 9,5\% foi devido à impossibilidade financeira (incapacidade de pagamento), 4,4\% propôs um valor menor que o sugerido; e $25,0 \%$ demonstrou ser um voto de protesto. A Tabela 2 apresenta a participação percentual e estatísticas descritivas para cada variável explicativa utilizada no modelo, segundo os tipos de respostas dos entrevistados. 
Tabela 2: Participação percentual e estatísticas descritivas das variáveis explicativas segundo o tipo de resposta do entrevistado para a valoração ambiental da área rural de Coxilha Rica/SC.

\begin{tabular}{|l|l|l|l|l|l|}
\hline \multirow{2}{*}{ Variável } & \multicolumn{2}{|l|}{ Resposta } & \multicolumn{2}{l|}{ Resposta: Não } \\
\cline { 2 - 6 } & $\begin{array}{l}\text { Sim } \\
(61,1 \%)\end{array}$ & $\begin{array}{l}\text { Não } \\
(38,9 \%)\end{array}$ & Limitação Financeira (9,5\%) & Sugeriu Preço Menor (4,4\%) & $\begin{array}{l}\text { Voto de Protesto } \\
(25,0 \%)\end{array}$ \\
\hline Conhece $(\%)$ & 89,6 & 89,8 & 42,5 & 81,8 & 90,5 \\
\hline Idade $(\mu ; \sigma)$ & $36,9(15,9)$ & $44,8(17,0)$ & $48,3(17,1)$ & $29,4(15,7)$ & $46,2(16,0)$ \\
\hline Educação $(\mu ; \mathrm{Mo})$ & $3,8(4)$ & $3,6(4)$ & $2,4(1)$ & $4,0(4)$ & $4,0(4)$ \\
\hline Renda $(\mu ; \mathrm{Mo})$ & $2,5(2)$ & $2,5(2)$ & $1,5(2)$ & $2,4(3)$ & $2,9(3)$ \\
\hline Pessoas $(\mu ; \mathrm{Mo})$ & $3,1(3)$ & $2,9(3)$ & $2,8(3)$ & $2,5(2)$ & $3,0(3)$ \\
\hline EPA $(\mu ; \sigma)$ & $61,0(6,4)$ & $57,5(6,4)$ & $54,5(7,6)$ & $60,3(7,0)$ & $58,1(6,3)$ \\
\hline Preço $(\mu ; \sigma)$ & $7,0(5,7)$ & $9,0(6,9)$ & $9,0(6,5)$ & $13,2(6,5)$ & $8,3(6,0)$ \\
\hline
\end{tabular}

Nota: $\mu$ : média; $\sigma:$ desvio padrão; Mo: moda.

A análise preliminar dos dados da Tabela 2 sugere que pessoas mais jovens estão mais predispostas à contribuição. Outras duas variáveis que indicam diferenças entre as respostas 'Sim' e 'Não' foram a EPA, maior para as respostas 'Sim', e o Preço, maior para as respostas 'Não'. Para as demais variáveis explicativas não se observou diferenças importantes. Chama atenção as estatísticas do grupo com limitação financeira, com os menores índice de renda, escolaridade, EPA e conhecimento do recurso ambiental estudado.

O maior preço sugerido está associado a resposta com sugestão de um preço menor. A medida que o preço sugerido pelo método referendo aumenta, diminui a participação percentual de respostas 'Sim' (Figura 1). Observa-se que mais de $70 \%$ demonstrou disponibilidade em contribuir com o menor valor sugerido ( $R \$ 1,00$ ), diminuindo linearmente para $40 \%$ para o maior valor ( $R \$ 20,00$ ). Nota-se um comportamento atípico para o valor sugerido de $\mathrm{R} \$ 15,00$, decorrente de comportamento aleatório da amostragem.

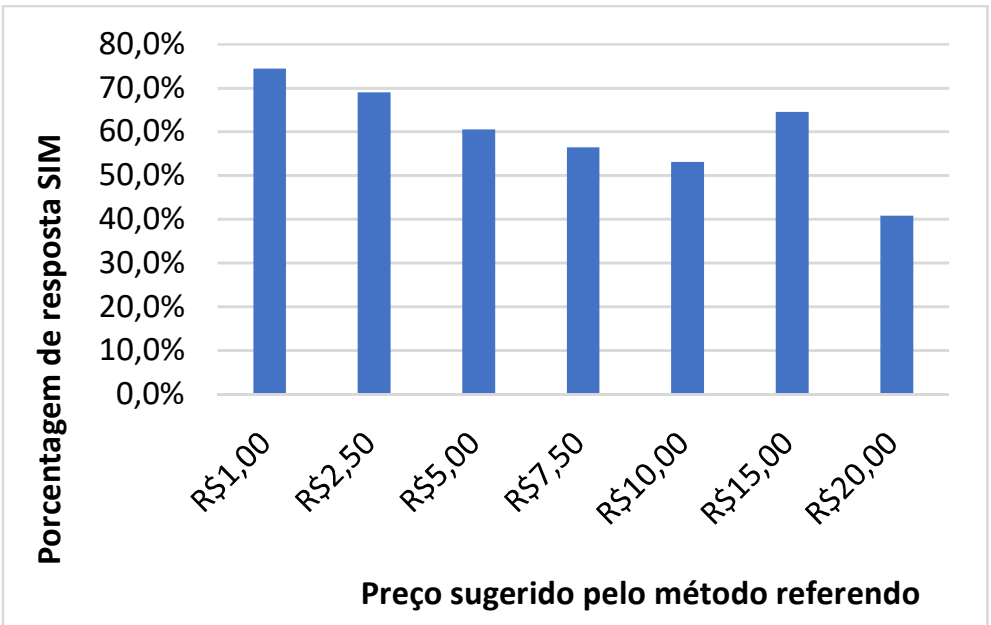

Figura 1: Porcentagem de respostas 'Sim' para cada valor sugerido pelo método referendo na valoração da área rural de Coxilha Rica - Lages/SC.

Considerando que grande parte dos entrevistados não usufruem diretamente dos benefícios do recurso ambiental estudado, pode-se considerar que o percentual de respostas positivas $(61,1 \%)$ é alto quando comparado a estudos similares. Simioni et al. (2015) encontraram $54,4 \%$ de respostas positivas para 
preservação do Salto Caveiras - área destinada ao turismo e lazer da população local. Resultados semelhantes foram encontrados por Silva et al. (2004), com 68\% de respostas positivas para preservação do Parque Chico Mendes (Rio Branco/AC), Cirino et al. (2008), de 59,8\% para a preservação da Área de Proteção Ambiental (APA) São José, em Minas Gerais, Obara (1999) obteve 72,9\% para a preservação da estação ecológica de Jataí (Luiz Antônio/SP), e por Sousa et al. (2006), com 63,4\% de respostas positivas para a preservação do Parque Metropolitano de Pituaçu (Salvador/BA).

A resposta "Não" para a contribuição foi classificada em dois motivos principais: à impossibilidade financeira ou a um voto de protesto. No primeiro caso, denota que o entrevistado não possui condições financeiras que lhe permita apresentar uma DAP positivas, configurando-se como uma justificativa real ou verdadeira e verificada em 9,5\% dos entrevistados. No caso dos votos de protesto ( $25 \%$ - 63 respondentes), as justificativas apresentadas foram: o governo deveria financiar este tipo de investimento (40,6\%); já existe uma alta carga tributária, e que esta deve incluir este tipo de atividade/ação (21,7\%); não acredita na efetivação da contribuição pela ONG $(14,5 \%)$; ou por outros motivos $(23,2 \%)$. No trabalho de Silva et al. (2004), o motivo mais citado foi: 'é um dever da prefeitura', revelando que o indivíduo não faz parte do processo de tomada de decisão na gestão dos recursos naturais, pois deposita toda a responsabilidade em assegurar a preservação ambiental sobre o poder público. Resultados semelhantes também foram encontrados por Souza et al. (2006), atribuindo a responsabilidade ao governo, à alta carga tributária e à desconfiança em organizações particulares de preservação da natureza.

Na presente pesquisa, como o recurso ambiental pesquisado se trata de área rural, merece destaque a alegação de que a responsabilidade para a preservação a Coxilha Rica é dos moradores da mesma. Os entrevistados relataram que não contemplam a localidade e, por esta razão, não se sentem parte dela para contribuir monetariamente, refletindo a influência do uso direto do bem avaliado nas respostas.

O nível de escolaridade mostrou-se significativo nos estudos de Oliveira et al. (2013), o qual afirma que pessoas com maior nível de escolaridade estão menos dispostas a contribuir. Como se espera, pessoas com baixo nível de escolaridade, indicam uma postura inferior de senso crítico e considerando um fato fictício de contribuição, aceitariam por acreditar que é o certo, e não por possuir senso de concepção ambiental. Diferentemente da minoria dos entrevistados com graduação, aptos de senso críticos que tendem a não contribuir para a manutenção do bem público, devido à alta carga tributária que já pagam e a presença de corrupção e desvio de dinheiro que atua no Brasil. Os resultados dos modelos de regressão logit e probit com os votos de protesto (Tabela 3 ) foram influenciados significativamente pelas variáveis Idade, Preço e EPA $(p<0,05)$.

A avaliação do poder preditivo dos modelos mostrou que os mesmos não são capazes de produzir estimativas confiáveis. Esta conclusão resulta da análise do valor relativamente alto para -2LL, sendo que quanto mais próximo de zero, maior é seu poder preditivo. Considerando também o $\mathrm{R}^{2}$ logit, houve uma melhoria na qualidade das predições para os modelos logit e probit, de apenas $10,32 \%$ e $10,28 \%$, respectivamente, com a inclusão das variáveis independentes. Um terceiro mecanismo para avaliar o grau de acurácia dos modelos é o teste de Hosmer-Lemeshow, cujo resultado indica que houve diferença 
significativa $(P<0,05)$ entre as predições realizadas pelo modelo e as observadas. Ainda, os modelos obtidos com a inclusão dos votos de protesto apresentam coeficientes negativos para as variáveis Educação e Renda, contrariando a hipótese inicial esperada.

Tabela 3: Coeficientes e indicadores de significância para a regressão binária logística (logit e probit) para a valoração da Coxilha Rica - Lages/SC.

\begin{tabular}{|c|c|c|c|c|c|c|}
\hline \multirow[t]{2}{*}{ Variável } & \multicolumn{3}{|c|}{ Logit $(n=252)$} & \multicolumn{3}{|c|}{ Probit $(n=252)$} \\
\hline & Coef. & Wald & $p$-value & Coef. & Wald & $p$-value \\
\hline Constante & $-3,0031$ & $-1,92$ & 0,055 & $-1,8279$ & $-1,93$ & 0,053 \\
\hline Conhece & $-0,0637$ & $-0,14$ & 0,890 & $-0,0289$ & $-0,10$ & 0,917 \\
\hline Idade & $-0,0232$ & $-2,39$ & 0,017 & $-0,0138$ & $-2,36$ & 0,018 \\
\hline Educação & $-0,0103$ & $-0,10$ & 0,919 & $-0,0023$ & $-0,04$ & 0,970 \\
\hline Renda & $-0,0809$ & $-0,52$ & 0,606 & $-0,0557$ & $-0,59$ & 0,557 \\
\hline Pessoas & 0,0467 & 0,14 & 0,887 & 0,0171 & 0,24 & 0,810 \\
\hline Preço & $-0,0604$ & $-2,57$ & 0,010 & $-0,0374$ & $-2,64$ & 0,008 \\
\hline EPA & 0,0864 & 3,71 & 0,000 & 0,0520 & 3,74 & 0,000 \\
\hline$-2 \mathrm{LL}$ & \multicolumn{3}{|c|}{$-151,015$} & \multicolumn{3}{|l|}{$-151,085$} \\
\hline $\mathrm{R}^{2}$ Logit & \multicolumn{3}{|l|}{0,1032} & \multicolumn{3}{|l|}{0,1028} \\
\hline Hosmer-Lemeshow & \multicolumn{3}{|c|}{$18,190(p<0,020)$} & \multicolumn{3}{|c|}{$15,702(p<0,047)$} \\
\hline
\end{tabular}

Considerando os modelos de regressão excluindo os votos de protesto da análise (Tabela 4), as variáveis significativas foram a Renda, o Preço e a EPA $(p<0,05)$. Nestes modelos, a confiabilidade na predição foi melhorada significativamente. Para ambas as funções, logit e probit, houve significativa redução do valor de $-2 \mathrm{LL}$, a introdução das variáveis explicativas melhorou as estimativas em cerca de $22 \%$ e o teste de Hosmer-Lemeshow não foi significativo $(P>0,05)$, indicando que não há diferença estatística entre os valores preditos e os observados e, portanto, os modelos produzem estimativas confiáveis.

Tabela 4: Coeficientes e indicadores de significância para a regressão binária logística (logit e probit) para a valoração da Coxilha Rica - Lages/SC, sem os votos de protestos.

\begin{tabular}{|c|c|c|c|c|c|c|}
\hline \multirow[t]{2}{*}{ Variável } & \multicolumn{3}{|c|}{ Logit $(n=189)$} & \multicolumn{3}{|c|}{ Probit (n=189) } \\
\hline & Coef. & Wald & $p$-value & Coef. & Wald & $p$-value \\
\hline Constante & $-5,2468$ & $-2,27$ & 0,023 & $-2,9198$ & $-2,25$ & 0,024 \\
\hline Conhece & $-0,0554$ & $-0,08$ & 0,936 & $-0,0508$ & $-0,13$ & 0,896 \\
\hline Idade & $-0,0032$ & $-0,22$ & 0,826 & $-0,0012$ & $-0,14$ & 0,886 \\
\hline Educação & 0,1576 & 0,93 & 0,352 & 0,0821 & 0,87 & 0,382 \\
\hline Renda & 0,8324 & 2,69 & 0,007 & 0,4403 & 2,66 & 0,008 \\
\hline Pessoas & 0,0377 & 0,22 & 0,825 & 0,0353 & 0,37 & 0,713 \\
\hline Preço & $-0,1126$ & $-3,29$ & 0,001 & $-0,0662$ & $-3,43$ & 0,001 \\
\hline EPA & 0,0940 & 2,83 & 0,005 & 0,0535 & 2,85 & 0,004 \\
\hline$-2 \mathrm{LL}$ & \multicolumn{3}{|l|}{$-70,447$} & \multicolumn{3}{|l|}{$-70,639$} \\
\hline $\mathrm{R}^{2}$ Logit & \multicolumn{3}{|l|}{0,2221} & \multicolumn{3}{|l|}{0,2200} \\
\hline Hosmer-Lemeshow & \multicolumn{3}{|c|}{$14,198(p<0,077)$} & \multicolumn{3}{|c|}{$7,245(p<0,510)$} \\
\hline
\end{tabular}

A alteração nos resultados obtidos com a exclusão dos votos de protesto também foi observada por Justo et al. (2014), que utilizaram a regressão logit para obter a DAP média para o Parque Ecológico Estadual do Sítio Fundão, no Ceará, e obtiveram como resposta os valores de $R \$ 3,33$ e $R \$ 7,77$ para os modelos com e sem votos de protesto, respectivamente.

As variáveis Conhece, Idade, Educação e Pessoas não foram significativas. Quanto as variáveis Conhece e Idade o efeito indicado pelos modelos foi negativo, contrariando a hipótese inicial. Paixão (2011) obteve coeficiente positivo para o conhecimento do recurso ambiental, porém assim como no presente trabalho a variável também não foi significativa. 
A variável Idade apresentou comportamento diverso: para Gomes (2013), o efeito foi negativo e não significativo; para Silveira et al. (2013), Cirino et al. (2008), Mattos et al. (2007) o coeficiente foi negativo e significativo; para Freitas et al. (2010) encontraram efeito positivo e não significativo; e Justo et al. (2014) também encontraram efeito positivo, porém, significativo. Tais resultados sugerem a variável Idade está sujeita às características predominantes da população estudada. Um aspecto importante a ser considerado são as ações de educação ambiental que são realizadas, sobretudo com jovens e no ambiente escolar, contribuindo para a obtenção de um coeficiente negativo, ou seja, pessoas mais jovens estariam mais propensas a contribuir positivamente.

Já as variáveis Educação e Pessoas apresentaram coeficiente positivo, tal como era esperado nas hipóteses iniciais, porém não foram significativas. Para a Educação, o comportamento da variável também foi diverso: efeito negativo e significativo em Oliveira et al. (2013); efeito positivo e não significativo em Freitas et al. (2010), Cirino et al. (2008), Mattos et al. (2007); e efeito positivo e significativo em Rodrigues et al. (2012), Justo et al. (2014). A variável número de pessoas na família normalmente não tem sido utilizada como variável explicativa nos estudos. Considerando somente as variáveis significativas do modelo sem os votos de protesto obteve-se o modelo final (Tabela 5).

Tabela 5: Coeficientes e indicadores de significância para a regressão binária logística (logit e probit) para a valoração da Coxilha Rica - Lages/SC, sem os votos de protestos e somente com as variáveis significativas.

\begin{tabular}{|l|l|l|l|l|l|l|}
\hline \multirow{2}{*}{ Variável } & Logit $(\mathrm{n}=189)$ & \multicolumn{1}{l|}{ Probit $(\mathrm{n}=189)$} \\
\cline { 2 - 7 } & Coef. & Wald & $\mathrm{p}$-value & Coef. & Wald & $\mathrm{p}$-value \\
\hline Constante & $-5,1583$ & $-2,72$ & 0,007 & $-2,8294$ & $-2,63$ & 0,009 \\
\hline Renda & 0,9543 & 3,38 & 0,001 & 0,5194 & 3,43 & 0,001 \\
\hline Preço & $-0,1104$ & $-3,29$ & 0,001 & $-0,0650$ & $-3,44$ & 0,001 \\
\hline EPA & 0,0954 & 2,89 & 0,004 & 0,0537 & 2,89 & 0,004 \\
\hline-2 LL & $-71,229$ & $-71,306$ & \\
\hline R Logit & 0,2135 & 0,2126 & $10,543(p<0,229)$ \\
\hline Hosmer-Lemeshow & $7,016(p<0,535)$ \\
\hline
\end{tabular}

O cálculo do valor ambiental individual mensal para a preservação da Coxilha Rica foi realizado utilizando-se o modelo 3, conforme segue no Quadro 1.

Quadro 1: Cálculo da Disposição a Pagar (DAP) mensal para a preservação da Coxilha Rica.

\begin{tabular}{|l|l|}
\hline Cálculo da DAP - modelo Logit & Cálculo da DAP - modelo Probit \\
\hline Benefício $=-5,1583+0,9543$ Renda -0,1104 Preço +0,0954 EPA & Benefício $=-2,8294+0,5194$ Renda $-0,0650$ Preço + 0,0537 EPA \\
0,1104 Preço $=-5,1583+0,9543$ Renda $+0,0954$ EPA & 0,0650 Preço $=-2,2948+0,5194$ Renda + 0,0537 EPA \\
Preço $=25,81$ & Preço $=25,17$ \\
\hline
\end{tabular}

A variável Renda apresentou coeficiente positivo e significativo $(P<0,05)$, confirmando a hipótese inicial do presente trabalho. Resultados semelhantes foram encontrados por Gomes (2013), Justo et al. (2014), Freitas et al. (2010), Rodrigues et al. (2012), Silveira et al. (2013), Cirino et al. (2008) e Mattos et al. (2007). Por outro lado, em Oliveira et al. (2013) e Paixão (2011) a renda foi positiva e não significativa, resultado atribuído à incapacidade de pagamento como a razão para uma DAP negativa, mas não influencia na decisão dos entrevistados. Hildebrand et al. (2002) destacam que mesmo em situações idênticas, quando avaliadas em locais com diferentes características culturais, econômicas e sociais podem levar a resultados 
bastante diferentes. Um quadro resumo dos coeficientes e significâncias das principais variáveis socioeconômicas é apresentado na Tabela 6.

Tabela 6: Coeficientes e significância das principais variáveis explicativas utilizadas nos Métodos de Valoração Contingente.

\begin{tabular}{|l|l|l|l|}
\hline \multirow{3}{*}{ Variáveis } & $\begin{array}{l}\text { Sinal do } \\
\text { Coeficiente }\end{array}$ & Significância & Referências \\
\hline Conhece & Positivo & Não & Paixão (2011) \\
\hline Idade & Negativo & Não & Gomes (2013) \\
\cline { 2 - 4 } & Negativo & Sim & Silveira et al. (2013), Cirino et al. (2008), Mattos et al. (2007) \\
\cline { 2 - 4 } & Positivo & Não & Freitas et al. (2010) \\
\cline { 2 - 4 } & Positivo & Sim & Justo et al. (2014) \\
\hline \multirow{3}{*}{ Educação } & Negativo & Sim & Oliveira et al. (2013) \\
\cline { 2 - 4 } & Positivo & Não & Freitas et al. (2010), Cirino et al. (2008), Mattos et al. (2007) \\
\cline { 2 - 5 } & Positivo & Sim & Rodrigues et al. (2012), Justo et al. (2014) \\
\hline \multirow{2}{*}{ Renda } & Positivo & Sim & $\begin{array}{l}\text { Gomes (2013), Justo et al. (2014), Freitas et al. (2010), Rodrigues et al. (2012), } \\
\text { Silveira et al. (2013), Cirino et al. (2008), Mattos et al. (2007) }\end{array}$ \\
\hline & & Oliveira et al. (2013), Paixão (2011) \\
\hline
\end{tabular}

Tabela 7: Participação percentual e medidas de tendência central da pontuação obtida por questão da Escala de Percepção Ambiental (EPA), para a amostra sem os votos de protesto.

\begin{tabular}{|c|c|c|c|c|c|c|c|c|}
\hline \multirow[t]{2}{*}{ № Questão } & \multicolumn{5}{|c|}{ Participação percentual por Pontuação } & \multicolumn{3}{|c|}{ Medidas de Tendência Central } \\
\hline & 1 & 2 & 3 & 4 & 5 & Média & Moda & Mediana \\
\hline 1 & 1,6 & 1,6 & 3,2 & 19,0 & 74,6 & 4,6 & 5,0 & 5,0 \\
\hline 2 & 6,9 & 6,9 & 3,2 & 20,1 & 63,0 & 4,3 & 5,0 & 5,0 \\
\hline 3 & 1,6 & 3,7 & 3,7 & 24,3 & 66,7 & 4,5 & 5,0 & 5,0 \\
\hline 4 & 4,8 & 3,2 & 0,5 & 23,3 & 68,3 & 4,5 & 5,0 & 5,0 \\
\hline 5 & 9,0 & 16,4 & 10,6 & 26,5 & 37,6 & 3,7 & 5,0 & 4,0 \\
\hline 6 & 6,9 & 4,8 & 4,2 & 18,5 & 65,6 & 4,3 & 5,0 & 5,0 \\
\hline 7 & 0,0 & 0,0 & 1,6 & 11,6 & 86,8 & 4,9 & 5,0 & 5,0 \\
\hline 8 & 4,8 & 5,3 & 21,2 & 19,6 & 49,2 & 4,0 & 5,0 & 4,0 \\
\hline 9 & 10,1 & 12,2 & 21,2 & 19,0 & 37,6 & 3,6 & 5,0 & 4,0 \\
\hline 10 & 1,1 & 0,5 & 0,5 & 12,7 & 85,2 & 4,8 & 5,0 & 5,0 \\
\hline 11 & 13,8 & 14,8 & 6,9 & 31,7 & 32,8 & 3,6 & 5,0 & 4,0 \\
\hline 12 & 38,6 & 25,4 & 15,9 & 8,5 & 11,6 & 2,3 & 1,0 & 2,0 \\
\hline 13 & 53,4 & 9,5 & 0,0 & 6,9 & 30,2 & 2,5 & 1,0 & 1,0 \\
\hline 14 & 7,9 & 1,6 & 2,1 & 25,9 & 62,4 & 4,3 & 5,0 & 5,0 \\
\hline 15 & 7,9 & 2,6 & 4,8 & 23,3 & 61,4 & 4,3 & 5,0 & 5,0 \\
\hline
\end{tabular}

Uma variável importante a ser considerada na valoração de recurso ambiental, uma vez que pode influenciar diretamente sobre a DAP, é o nível de consciência ou percepção ambiental do entrevistado. Neste trabalho, a percepção ambiental foi avaliada por uma escala (EPA) que se mostrou significativa nos resultados dos modelos de regressão. Como medida de confiabilidade das respostas da escala EPA, os dados foram submetidos ao teste de alfa de Cronbach (1951), obtendo-se o valor de 0,5619, bem próximo ao encontrado por Simioni et al. (2015), de 0,5950 e superior ao de Queiroga (2006), de 0,51 para consumidor socialmente responsável. Todavia, retirando-se as questões 12 e 13, o valor do alfa se elava para 0,5859 e 0,5976, respectivamente. Também, quando se remove as duas questões simultaneamente, o valor alfa de Cronbach aumenta para 0,6224. Diante disso, pode inferir que os problemas que ocorrem no Brasil, como a corrupção e a debilidade do transporte público, são fatores que interferiram negativamente nos resultados. Uma análise mais apurada das respostas considerando a amostra sem os votos de protesto é apresentada na Tabela 7.

Os dados evidenciam que os entrevistados concordam que as organizações ambientais atuais estão mais interessadas em incomodar a sociedade do que lutar contra a poluição (65\% obtiveram 1 ou 2 pontos) 
e que mesmo que o transporte público gere menos poluição, utilizam carro ou moto $(53,4 \%$ obtiveram 1 ponto). De acordo com Silveira et al. (2013), um dos principais problemas e mais recorrentes que afeta as cidades brasileiras, é a precariedade do transporte público coletivo.

\section{CONCLUSÕES}

É importante considerar que o objetivo inicial deste trabalho foi a valoração ambiental da área rural de Coxilha Rica (SC), considerando a preservação da paisagem típica e do patrimônio histórico-cultural representado pelo caminho das tropas. Portanto, representa o custo de oportunidade social da não preservação desses recursos.

O método adotado para estimar o valor econômico - MVC, mostrou-se satisfatório, embora dois fatores críticos merecem destaque: a baixa escolaridade dos entrevistados e o atual ambiente econômico e político brasileiro. A baixa escolaridade constituiu-se um fator limitante na medida em que restringiu a compreensão dos questionamentos e objetivos da pesquisa, sobretudo nas questões relacionadas a EPA, motivo pelo qual a variável Educação mostrou-se não significativa nos resultados dos modelos. No que tange à realidade brasileira, o cenário de corrupção, crise econômica e elevada carga tributária, associados à baixa eficiência do uso dos recursos públicos impactaram em elevados votos de protesto. Nesse aspecto, as pessoas com maior nível de escolaridade evidenciaram maior senso crítico quanto à realidade brasileira, contribuindo também para estimativa mais conservadora ou para votos de protesto.

A relação entre renda e DAP se mostrou significativa dado o relativo grau de privacidade do bem avaliado, ou seja, pelo fato de se tratar de uma área rural configura o bem como privado. Nesse sentido, a apreciação da paisagem e da estrutura remanescente do cominho das tropas é possível de ser realizada mediante acompanhamento de guia turístico e com estrutura de transporte adequada, dada a distância da cidade e das condições das vias públicas na região, configurando-se o fator financeiro como limitante.

Pode-se concluir ainda que a EPA se apresentou como medida importante e viável a ser utilizada nos modelos econométricos como variável explicativa na estimativa da DAP dos indivíduos. A EPA se associa, de uma certa forma, com a predisposição das pessoas em contribuir financeiramente, na medida em que reflete maior consciência e/ou percepção do entrevistado para as questões ambientais de maneira geral.

Os modelos logit e probit para preservação da Coxilha Rica resultaram em estimativas significativamente mais confiáveis quando foram retirados os votos de protesto das análises. Estes resultados evidenciam a influência negativa dos votos de protestos nos resultados das análises, configurando-se como um importante viés estatístico que requer um tratamento adequado. Os valores anuais obtidos foram de R\$ 49.127.786,40 e R\$ $47.909 .584,80$, respectivamente, para os modelos logit e probit. Esse valor representa economicamente, a disposição da população de Lages/SC em manter preservadas as paisagens típicas de campo nativo com araucárias, bem como os recursos histórico-culturais relacionados aos caminhos das tropas nela contidos. Como é inerente ao método, trata-se de uma taxa hipotética e representa o anseio de manter vivo um dos símbolos que identifica a região serrana. 


\section{REFERÊNCIAS}

ARROW, K. J.; SOLOW, R.; PORTNEY, P. R.; LEAMER, E. E.; RADNER, R.; SCHUMAN, H.. Report of the N.O.A.A. on contingent valuation. Federal Register, v.58, n.10, p.46014614, 1993

BOLSAN, R.. Impactos decorrentes da modernização da agricultura brasileira. CAMPO-TERRITÓRIO: revista de geografia agrária, v.1, n.2, p.123-151, 2006.

CIRINO, J. F.; LIMA, J. E.. Valoração contingente da Área de Proteção Ambiental (APA) São José - MG: um estudo de caso. Revista de Economia e Sociologia Rural, v.46, n.3, p.647-672, 2008. DOI: http://dx.doi.org/10.1590/S0103$\underline{20032008000300004}$

CRONBACH, L. J.. Coefficient alpha and the internal structure of tests. Psychometrica, v.16, p.297-334, 1951.

DESVOUSGES, W.; MATHEWS, K.; TRAIN, K.. Adequate responsiveness to scope in contingent valuation. Ecological Economics, v.84, p.121-128, 2012. DOI: https://doi.org/10.1016/j.ecolecon.2012.10.003

FREITAS, K. A. A.; BARBOSA FILHO, J.; PIO, N. S.; SILVA, F. F.; MORAES, L. S.. Valoração econômica dos benefícios ambientais percebidos pela população da bacia do Educandos provenientes do PROSAMIM. Acta Amazônica, v.40, n.3, p.509-514, 2010. DOI:

http://dx.doi.org/10.1590/S0044-59672010000300009

GARCÍA, M. C.. La ambientalización de la Universidad: un estudio sobre la formación ambiental de los estudiantes de la universidad de Santiago de Compostela y la política ambiental de la institución. Tese (Doctorado in Educación) Universidad de Santiago de Compostela, Santiago de Compostela, 2000.

GOMES, I. D.. Crescimento económico em Cabo Verde e seu impacto na sustentabilidade ambiental: aplicação do método de avaliação contingente, através da técnica disposição a pagar para a valoração ambiental. Dissertação (Mestrado em Economia e Gestão de Ambiente) - Faculdade de Economia da Universidade do Porto, Porto, 2013.

GULLO, M. C. R.. Valoração econômica dos recursos naturais: uma aplicação para o setor industrial de Caxias do Sul. Tese (Doutorado em Economia) - Universidade Federal do Rio Grande do Sul, Porto Alegre, 2010.

HILDEBRAND, E.; GRAÇA, L. R.; HOEFLICH, V. A.. 'Valoração Contingente' na avaliação econômica de áreas verdes urbanas. Revista Floresta, v.32, n.1, p.121-132, 2002. DOI: http://dx.doi.org/10.5380/rf.v32i1.2353

IBGE. Cidades. Brasília: IBGE, 2016.

JUSTO, W. R.; RODRIGUES, C. P. B.. Valoração econômica do Parque Ecológico Estadual do Sítio Fundão, Crato, CE. Revista de Política Agrícola, n.1, p.4-17, 2014.

IPHAN conclui mapeamento do patrimônio cultural da Coxilha. Correio Lageano, Lages, 22 jun 2012.

LO, A.Y.; JIM, C.Y.. Protest response and willingness to pay for culturally significant urban trees: Implications for Contingent Valuation Method. Ecological Economics, v.114, p.58-66, 2015. DOI:

https://doi.org/10.1016/j.ecolecon.2015.03.012

MAJUMDAR, S.; DENG, J.; ZHANG, Y.; PIERSKALLA, C.. Using contingent valuation to estimate the willingness of tourists to pay for urban forests: a study in Savannah, Georgia. Urban Forestry \& Urban Greening, v.10, n.4, p.275-280, 2011. DOI: https://doi.org/10.1016/j.ufug.2011.07.006

MATTOS, A. D. M.; JACOVINE, L. A. G.; VALVERDE, S. R.; SOUZA, A. L. de; SILVA, M. L. da; LIMA, J. E.. Valoração ambiental de áreas de preservação permanente da microbacia do Ribeirão São Bartolomeu no município de Viçosa, MG. Revista Árvore, v.31, n.2, p.347-353, 2007. DOI: http://dx.doi.org/10.1590/S0100-67622007000200018

MINITAB. Minitab Statistical Software, Release $\mathbf{1 6}$ for Windows. Pennsylvania: State College, 2009.

MITCHELL, R. C.; CARSON, R. T.. Using surveys to value public goods: the contingent valuation method. Washington, DC: Resources for the Future, 1989.

MOTTER, A. F. C.. Colonização europeia no nw do Rio Grande do Sul: primeiros sinais de desequilíbrios ambientais. Mercator, v.14, n.1, p.105-112, 2015. DOI: http://doi.org/10.4215/RM2015.1401.0007

OBARA, A. T.. Valoração econômica de unidades de conservação: o método de valoração contingente. Caso de estudo: Estação Ecológica de Jataí (Luiz Antônio - SP). Tese (Doutorado em Ecologia e Recursos Naturais) - Universidade Federal de São Carlos, São Carlos, 1999.

OLIVEIRA, K. T. L. L.; MATA, H. T. C.. Qual o valor de uma praia limpa? Uma aplicação do método de valoração contingente no bairro Rio Vermelho, Salvador/BA. In: ENCONTRO DE ECONOMIA BAIANA, 9. Anais. Salvador: UFB/SEI, 2013.

PAIXÃO, A. G.. A valoração econômica da lagoa da Princesa: nascente do Rio Paraguai localizada no médio norte matogrossense. Dissertação (Mestrado em Economia) Universidade Federal de Mato Grosso, Cuiabá, 2011.

POLÊSE, C.. Coxilha Rica: subsídios a uma proposta de conservação para o sul do município de Lages - SC. Dissertação (Mestrado em Planejamento Territorial e Desenvolvimento Socioambiental) - Universidade do Estado de Santa Catarina, Florianópolis, 2014.

POLÊSE, C.; OLIVEIRA, F. H.; LIMA, C. L.; ALVES, F. E. Análise da hidrografia da Coxilha Rica, Sul do município de Lages SC. Geosul, v.30, n.59, p.47-66, 2015. DOI:

http://dx.doi.org/10.5007/2177-5230.2013v30n59p47

PUGAS, M. A. R. Valoração contingente de unidades de conservação: avaliando a DAP espontânea e induzida da população de Rondonópolis (MT) pelo horto florestal. Dissertação (Mestrado em Gestão Econômica do Meio Ambiente) - Universidade de Brasília, Brasília, 2006.

QUEIROGA, F.; GOUVEA, V. V.; COUTINHO, M. P. L.; PESSOA, V.; MEIRA, M.. Intenção de comportamento socialmente responsável do consumidor: sua relação com os valores 
humanos básicos. Psico-USF, v.11, p.239-248, 2006. DOI: http://dx.doi.org/10.1590/S1413-82712006000200012

RODRIGUES, W.; SANTANA, W. C.. Análise econômica de sistemas de gestão de resíduos sólidos urbanos: o caso da coleta de lixo seletiva em Palmas, TO. Revista Brasileira de Gestão Urbana, v.4, n.2, p.299-312, 2012. DOI: http://dx.doi.org/10.7213/urbe.7402

SILVA, E. M.; SILVA, E. M.; GONÇALVES, V.; MUROLO, A. C.. Estatística: para os cursos de economia, administração e ciências contábeis. São Paulo: Atlas, 1997.

SILVA, R. G.; LIMA, J. E.. Valoração contingente do parque 'Chico Mendes': uma aplicação probabilística do método referendum com bidding games. Revista de Economia e Sociologia Rural, v.42, n.4, p.685-708, 2004. DOI: http://dx.doi.org/10.1590/S0103-20032004000400008

SILVEIRA, V. C.; CIRINO, J. F.; PRADO FILHO, J. F.. Valoração econômica da área de proteção ambiental estadual da Cachoeira das Andorinhas - MG. Revista Árvore, v.37, n.2, p.257-266, 2013a. DOI: http://dx.doi.org/10.1590/S0100$\underline{67622013000200007}$
SILVEIRA, M. R.; COCCO, R. G.. Transporte público, mobilidade e planejamento urbano: contradições essenciais. Estudos Avançados, v.27, n.79, p.41-53, 2013b. DOI: http://dx.doi.org/10.1590/S0103-40142013000300004

SIMIONI, F.J.; MOMBACH, G. N. N.; ALVARENGA, R. A. F. de; DONADEL, C.; NASCIMENTO, A. C.; SOUZA, S. J.. Escala de consciência ambiental e análise multivariada na análise do método de valoração contingente do Salto Caveiras de Lages/SC. In: SEMINÁRIO DE INICIAÇÃO CIENTÍFICA, 25. Anais. Lages: UDESC, 2015.

SOUZA, G. B.; MOTA, J. A.. Valoração econômica de áreas de recreação: o caso do Parque Metropolitano de Pituaçu, Salvador, BA. Revista de Economia, v.32, n.1, p.37-55, 2006. DOI: http://dx.doi.org/10.5380/re.v32i1.6826

TEIXEIRA, J. C.. Modernização da agricultura no Brasil: impactos econômicos, sociais e ambientais. Revista Eletrônica da Associação dos Geógrafos Brasileiros, v.2, n.2, p.21-42, 2005.

TREKKING caminho das tropas. Correio Lageano, Lages, 21 mai 2013

A CBPC - Companhia Brasileira de Produção Científica (CNPJ: 11.221.422/0001-03) detém os direitos materiais desta publicação. Os direitos referem-se à publicação do trabalho em qualquer parte do mundo, incluindo os direitos às renovações, expansões e disseminações da contribuição, bem como outros direitos subsidiários. Todos os trabalhos publicados eletronicamente poderão posteriormente ser publicados em coletâneas impressas sob coordenação da Sustenere Publishing, da Companhia Brasileira de Produção Científica e seus parceiros autorizados. Os (as) autores (as) preservam os direitos autorais, mas não têm permissão para a publicação da contribuição em outro meio, impresso ou digital, em português ou em tradução. 